\title{
P013
}

\section{OPTIMIZATION OF TRICHROMATIC WHITE LED SPECTRUM FOR DIM LIGHTING CONDITIONS}

\author{
Hung-Chung Li et al.
}

DOI 10.25039/x47.2020.PO13

\section{Paper accepted for the $5^{\text {th }}$ CIE Symposium on Colour and Visual Appearance}

The paper was selected by the International Scientific Committee (ISC) for presentation at the 5th CIE Symposium on Colour and Visual Appearance, Hong Kong, CN, April 21-22, 2020, which, due to the corona pandemic, could not take place. The paper has not been peer-reviewed by CIE.

\section{(C) CIE 2020}

All rights reserved. Unless otherwise specified, no part of this publication may be reproduced or utilized in any form or by any means, electronic or mechanical, including photocopying and microfilm, without permission in writing from CIE Central Bureau at the address below. Any mention of organizations or products does not imply endorsement by the CIE.

This paper is made available open access for individual use. However, in all other cases all rights are reserved unless explicit permission is sought from and given by the CIE.

CIE Central Bureau

Babenbergerstrasse 9

A-1010 Vienna

Austria

Tel.: +4317143187

e-mail: ciecb@cie.co.at

www.cie.co.at 
P013

\title{
OPTIMIZATION OF TRICHROMATIC WHITE LED SPECTRA FOR DIM LIGHTING CONDITION
}

\author{
Li, H.C. ${ }^{1}$, Sun, P.L. ${ }^{2}$, Huang, Y.N. ${ }^{1}$ \\ ${ }^{1}$ Research Center for Information Technology Innovation, Academia Sinica, Taipei, CHINESE \\ TAIPEI, ${ }^{2}$ National Taiwan University of Science and Technology, Taipei, CHINESE TAIPEI
}

pony780210@citi.sinica.edu.tw

\begin{abstract}
Most night-time outdoor and traffic lighting scenarios are in the mesopic range. Because colour gamut in the mesopic conditions is relatively narrow, the general colour rendering index is not suitable for evaluating colour fidelity. Therefore, an optimization of the white LED spectrum based on 3D colour gamut is essential to raise the ability of colour recognition under constant contrast condition for the lighting application. The optimal trichromatic white LED spectra can be obtained by investigating the variance of wavelength positions and peak power density ratios for $10 \mathrm{~cd} / \mathrm{m}^{2}, 1 \mathrm{~cd} / \mathrm{m}^{2}, 0.3 \mathrm{~cd} / \mathrm{m}^{2}$, and $0.1 \mathrm{~cd} / \mathrm{m}^{2}$. As a result, a trade-off between mesopic luminance and colour gamut is quite significant. According to the predicted photopic/mesopic luminance level and the gamut volume, the test spectra with the highest Lmes, largest gamut volume (GV), and highest (Lmes+GV) are suggested as the optimal white LED spectra under a specific luminance level.
\end{abstract}

Keywords: Mesopic Vision, White LED, Spectral Optimization, Colour Gamut Estimation

\section{Introduction}

The luminance level of night-time conditions regularly falls into a mesopic visual range within $0.001 \mathrm{~cd} / \mathrm{m}^{2}$ to $3 \mathrm{~cd} / \mathrm{m}^{2}$. In the early stages of mesopic visual performance research, two methods, which were brightness matching and visual performance-based approaches, were mainly used for establishing the mesopic sensitivity (Eloholma, 2005). The mesopic spectral luminous efficiency could be described with a linear combination of the photopic $V(\lambda)$ and scotopic $V^{\prime}(\lambda)$ spectral luminous efficiency functions. From the previous studies, the design of the white LED spectrum considered the luminance and colour rendering index was solely in the photopic condition where the visual response of humanity was slightly different from the mesopic range. As a result, most researchers have aimed at proposing an appropriate spectrum for mesopic vision currently to meet the visual requirements based on mesopic photometry.

In the mesopic vision, models of mesopic luminous efficiency, such as X-model (Rea, 2004) and MOVE-model (Eloholma, 2006), were derived for calculating mesopic luminance (denoted as Lmes). The X-model was obtained from a reaction time experiment in the mesopic range. In the $X$-model, a parameter $X$ was used to characterize the ratio between photopic and scotopic luminous efficacy at any luminance. The MOVE-model was proposed by European research consortium MOVE (Mesopic Optimization of Visual Efficiency) as a recommended model for mesopic luminance of road lighting applications. The model was derived from a series of experiments related to visual task performance, such as achromatic contrast threshold, reaction time, and recognition threshold. Despite the similar forms of the X-model and MOVE-model, the major differences of the two models were that the upper luminance limit of the mesopic range, which was considered too low for the X-model and too high for the MOVE-model. Therefore, a modified MOVE-model was later proposed with an appropriate upper luminance limit to meet the street and road lighting conditions (Halonen, 2010). In 2008, Viikari et al. tested the X-model, MOVE-model, and modified MOVE-model by use of visual data acquired from European universities. As a result, the modified MOVE-model described the data best with over half of the situations (Viikari, 2008). 
To calculate mesopic luminance, the S/P-ratio of a light source was essential. It was the scotopic lumens divided by photopic lumens as Equation 1 where $k_{s}$ and $k_{p}$ were 1699 and 683 , respectively, which were the peak value of luminous efficiency in photopic and scotopic ranges. The same light source would have different values for photopic and scotopic lumens because the two conditions' response curves were different. In general, a light source with a higher correlated colour temperature was provided with a higher S/P-ratio (Zan, 2016). If a light source had a higher S/P-ratio, the visual brightness would be relatively higher in the scotopic conditions and likely enhance night-time visual performance and be beneficial to mesopic design (CIE191, 2010). However, if a light source had stronger spectral radiance in about $530 \mathrm{~nm}$, it should be relatively brighter in mesopic vision, but S/P-ratio could not depict it.

$$
S / P=\frac{L_{s}}{L_{p}}=\frac{\int_{400}^{750} k_{s} V^{\prime}(\lambda) S(\lambda) d \lambda}{\int_{400}^{750} k_{p} V(\lambda) S(\lambda) d \lambda}
$$

Shin et al. (Shin, 2004) proposed a mesopic colour appearance model to simulate the variation of colour appearance in the mesopic range and photopic. By adding rod intrusion to the two-stage model (Kaiser, 1996), the mesopic model describes that the opponent process converted the cone responses from L, M, and $S$ to red/green, yellow/blue opponent-colour and luminance channels denoted by $L-2 M, L+M-S$, and $L+M$, respectively. The model considered the characteristic of the nonlinear shift in spectral luminous efficiency, reduction of saturation at low illuminance levels, and the variations of hue and chroma loci under different illuminance levels from $1000 \mathrm{Ix}$ to $0.01 \mathrm{Ix}$. The experimental results showed that chroma decreases continuously with the decrease of the illuminance level until 0.01 Ix. However, it didn't take some complex visual phenomena such as Hunt effect, chromatic adaptation, and background luminous contrast into account.

A comparison of optimal trichromatic and tetrachromatic white LED spectra suggested by Lei et al. and Zan et al. was recently discussed ( $\mathrm{Li}, 2018)$. The results showed that the performance of both tetrachromatic white LEDs in mesopic luminance and colour gamut volume were not that ideal as those of the trichromatic white LED spectrum. Therefore, the optimization of trichromatic white LED spectra needs an intensive study to achieve the best result.

\section{Methods}

The approach of trichromatic white LED spectrum optimization is shown in Figure 1. The optimal white LED spectral power distribution (SPDs) for specific luminance level including $10 \mathrm{~cd} / \mathrm{m}^{2}, 1 \mathrm{~cd} / \mathrm{m}^{2}, 0.3 \mathrm{~cd} / \mathrm{m}^{2}$ and $0.1 \mathrm{~cd} / \mathrm{m}^{2}$ can be obtained by the following steps: First, individually generate SPDs of trichromatic white LED with 50000 times based on the data of Philips Color Blast measured by a spectroradiometer (Topcon SR-UL1R) and simulated Gaussian-like function as Equation 2 where $x$ and alpha represent the peak wavelength and a function of spectral half-width for each component respectively to establish a spectral database. $n$ is set as 2 in the study. The Full Width at Half Maximum (FWHM) of red, green, and blue LEDs are $15 \mathrm{~nm}, 40 \mathrm{~nm}, 25 \mathrm{~nm}$ for Philips Color Blast and $26 \mathrm{~nm}, 36 \mathrm{~nm}, 14 \mathrm{~nm}$ for simulated Gaussian-like function respectively. The trichromatic white LED spectra which the Duv are below 0.02, and the correlated colour temperature (CCT) between $1600 \mathrm{~K}$ and $10000 \mathrm{~K}$ are selected with the random shifts within $50 \mathrm{~nm}$ and ratios from 0.1 to 1 for red, green and blue LED as the test data. Totally 7929 and 4811 test SPDs of Philips Color Blast and simulated Gaussian-like function are collected. For consistency, we initialize the peak wavelength of red, green, and blue LED to $600 \mathrm{~nm}, 520 \mathrm{~nm}, 430 \mathrm{~nm}$. Second, apply an equalenergy white spectrum and input a specific luminance level to calculate the total radiance of the spectrum in $400 \mathrm{~nm}$ to $700 \mathrm{~nm}$ range. If the luminance level is below $5 \mathrm{~cd} / \mathrm{m}^{2}$, the modified MOVE-model is adopted to calculate the power of the spectrum. Conversely, only the photopic luminosity function is used. Third, input a series of test SPDs and acquire the absolute radiant power based on the same total radiance with the equal-energy white spectrum to calculate the photopic, scotopic, and mesopic luminance. Judd modified tristimulus values $X_{J u d d}, Y_{J u d d}, Z_{J u d d}$, and $\operatorname{CIE~} V^{\prime}(\lambda)$ are obtained by the integral of test spectra, 
spectral reflectance, Judd modified colour matching functions, and the scotopic luminous function. Then the tristimulus values are converted to the spectral responses of the cones, $\mathrm{L}$, $M$ and $S$. The scotopic luminance $Y^{\prime}$ is also calculated. Next, the $L, M$, and $S$ responses and luminance $Y^{\prime}$ are regarded as the inputs for the Shin's mesopic colour appearance model. Based on the experimental results from Shin's study, the weighting coefficients under a specific luminance level can be calculated with an interpolation process and divided by a solid angle between the range of $1000 \mathrm{Ix}$ and $0.01 \mathrm{Ix}$. The mesopic/photopic luminance acquired from test SPDs should be converted to the illuminance unit first for using the mesopic colour appearance model.

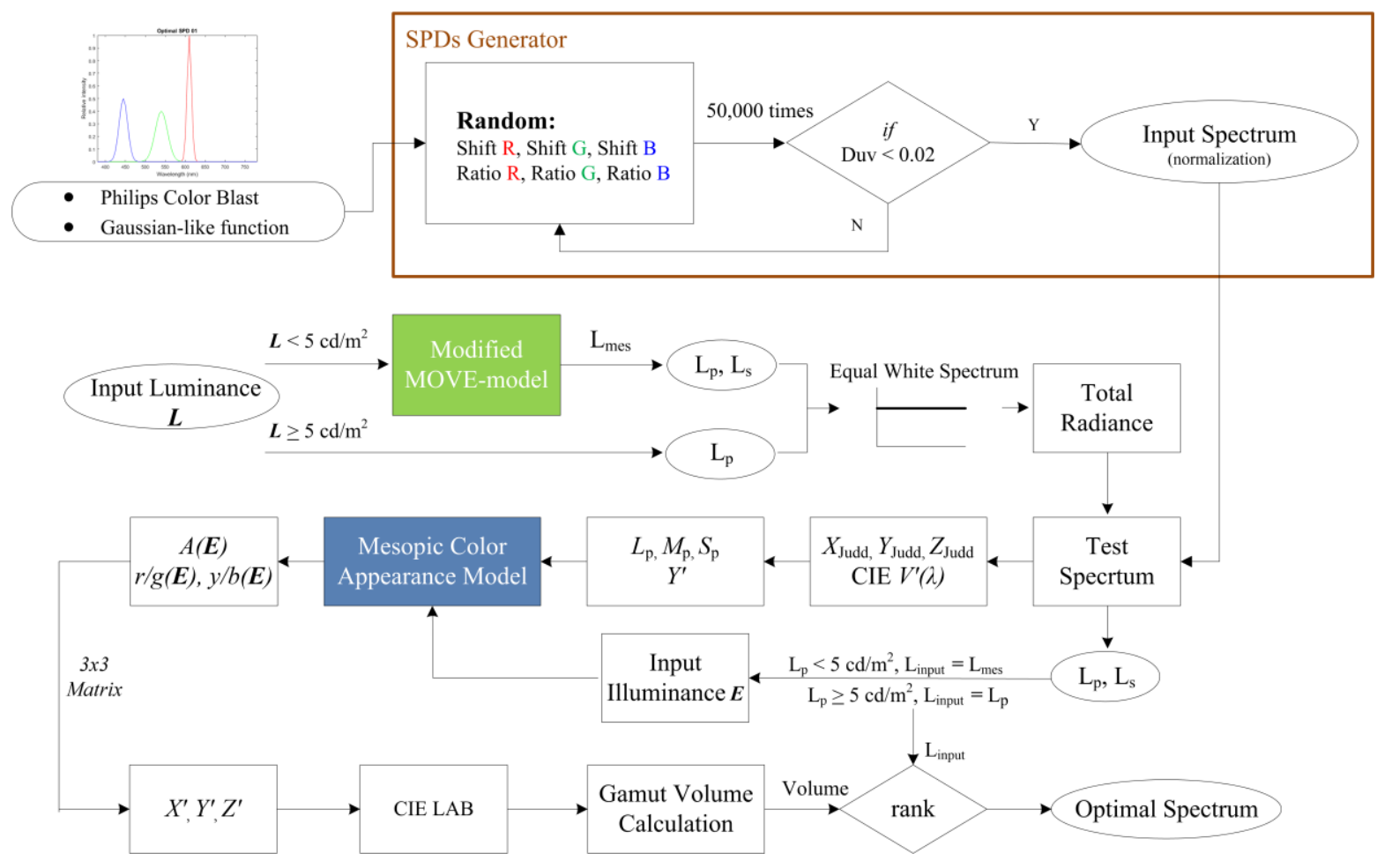

Figure 1 - Flow chart of trichromatic white LED spectrum optimization

$$
\operatorname{SuperGaussian}(x)=\exp \left(\frac{-0.5 \cdot a b s(x)^{n}}{a l p h a^{n}}\right), \text { alpha }=\mathrm{FWHM} \cdot \frac{\sqrt{2 \ln (2)}}{2}
$$

After simulating the colour appearance $A(E), r / g(E), y / b(E)$ under a specific luminance level, the simulated tristimulus values $X^{\prime}, Y^{\prime}, Z^{\prime}$ were obtained by a $3 \times 3$ transformation matrix and converted to CIE LAB colour space for its gamut volume calculation. The gamut volume is the summation of all tetrahedron's volume where the tetrahedra are determined by 3D Delaunay triangulation of the colour samples, and the unit of gamut volume is LAB cube. Finally, according to the predicted photopic/mesopic luminance level and the gamut volume, the test spectra with the highest $L_{\text {mes, }}$ largest gamut volume (GV), and highest ( $\left.L_{\text {mes }}+G V\right)$ are selected as the optimal white LED spectra under a specific luminance level.

\section{Results}

\subsection{Characteristics of trichromatic white LED spectra}

To better determine the characteristic of the trichromatic white LED spectrum on mesopic luminance and gamut volume, the spectra with the variance of peak wavelength shifts and the different powers of three components are investigated under $10 \mathrm{~cd} / \mathrm{m}^{2}$ and $1 \mathrm{~cd} / \mathrm{m}^{2}$ luminance levels. Note that the luminance level refers to the luminance of an equal energy white. The test SPDs are normalized to have the same total radiance to the equal energy white. Due to 
the similar characteristic of the simulated Gaussian-like function dataset, only the results of Philips Color Blast are present. As shown in Figure 2, the test spectrum where the blue, green, red LED located at $430 \mathrm{~nm}, 520 \mathrm{~nm}, 600 \mathrm{~nm}$ are shifted to $480 \mathrm{~nm}, 570 \mathrm{~nm}, 650 \mathrm{~nm}$ with $5 \mathrm{~nm}$ spectral interval.

The results indicate that the mesopic luminance would increase with the shifts of blue LED from the shorter wavelength to the longer one, but the maximum gamut volume could be achieved at a particular wavelength position under these two luminance levels. With the shifts of green LED, the gamut volume decreases acutely, but the highest mesopic luminance appears. However, the performance of mesopic luminance in the aspect of the red LED is entirely different from that of the blue LED. Besides, the two lighting levels show an identical tendency. The impacts of the RGB-LED ratio (i.e., the rate of the maximum powers of the blue, green and red LED) on mesopic luminance and gamut volume are also studied, and the results are shown in Figure 3 where the mesopic luminance increases with a higher ratio of green and red LED. By providing higher powers of blue and red LED can extend the range of colour gamut volume.
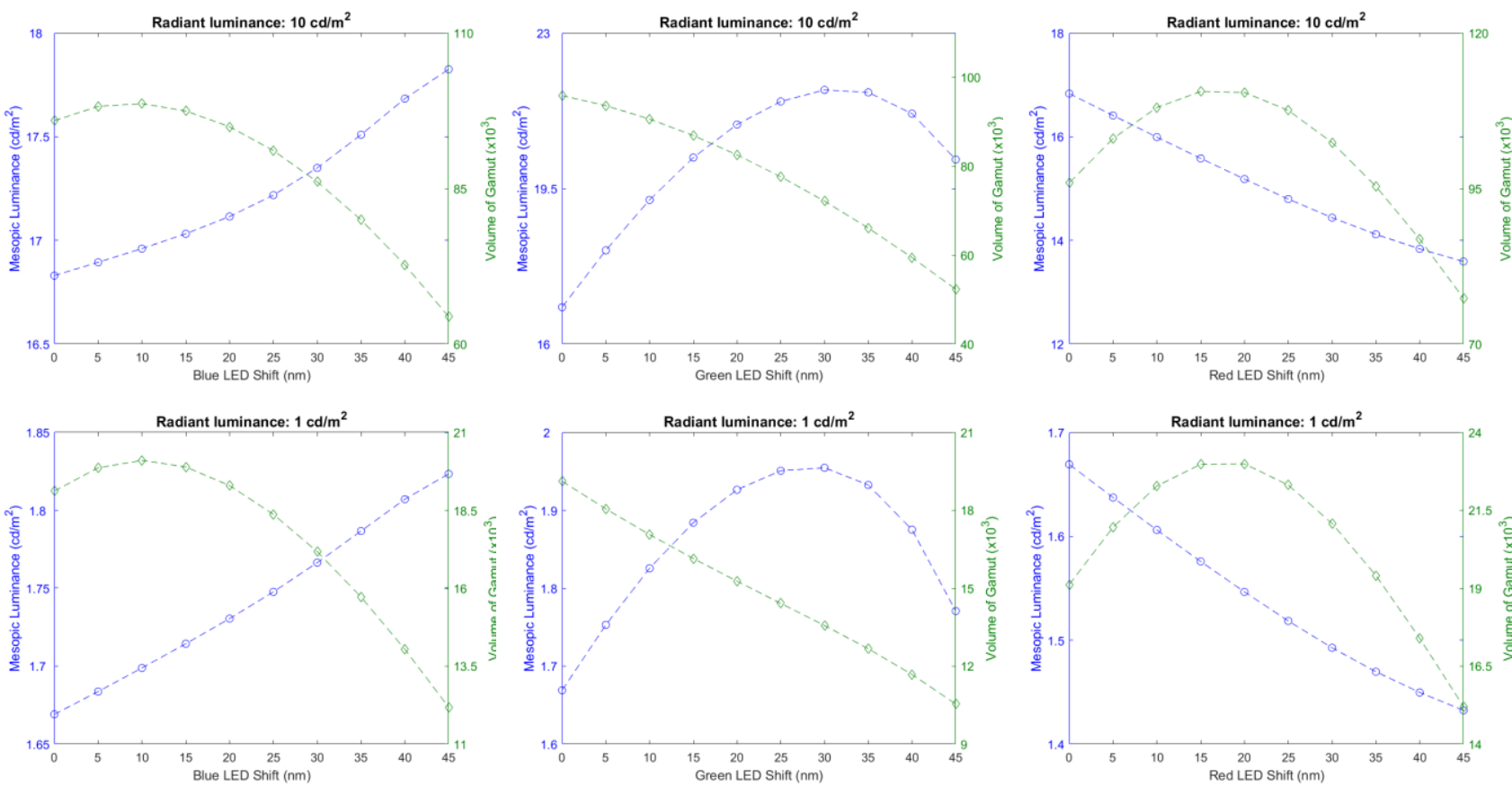

Figure 2 - Characteristic of luminance and gamut volume when shifting peak wavelength of LEDs (left to right: Blue, Green, and Red) 

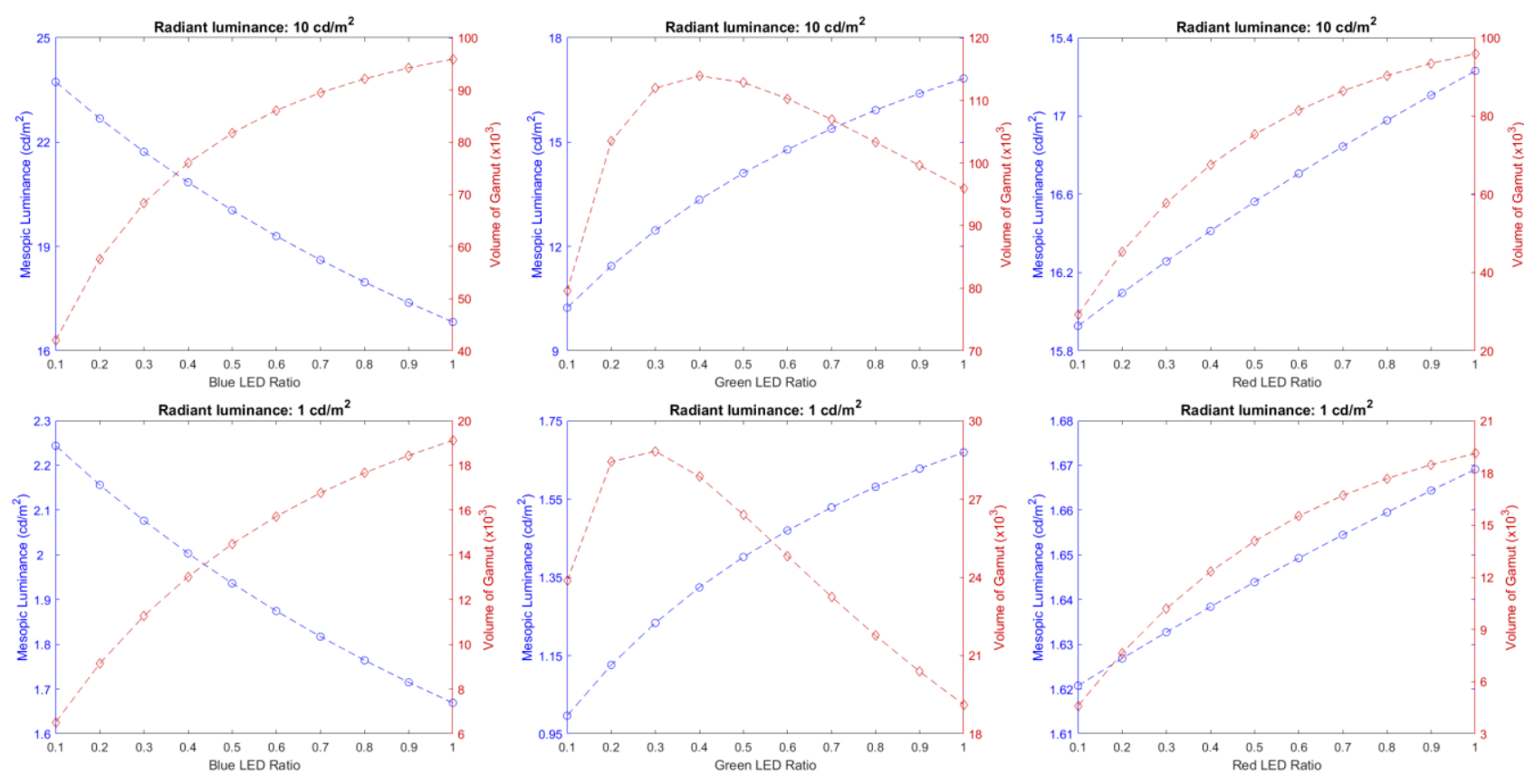

Figure 3 - Characteristic of luminance and gamut volume when changing the ratios of LEDs (left to right: Blue, Green, and Red)

\subsection{Optimal trichromatic white LED spectra}

Based on the white LED optimization method mentioned above, the optimal trichromatic white LED spectra are derived for $10 \mathrm{~cd} / \mathrm{m}^{2}, 1 \mathrm{~cd} / \mathrm{m}^{2}, 0.3 \mathrm{~cd} / \mathrm{m}^{2}$, and $0.1 \mathrm{~cd} / \mathrm{m}^{2}$ luminance levels, respectively. Due to the consideration of the constraint on computing, 3000 kinds of test SPDs for each dataset are tested in this study. In the theoretical simulation, the spectral reflectance of 1269 matt Munsell colour chips and the mesopic colour appearance model are used to optimize the white LED spectrum. The different shifts and ratios of red, green, and blue LED are the variables to simulate their gamut volumes in the same radiant quantity condition. The 24 optimal spectra and a colour gamut of the spectrum, which simultaneously provide relatively higher $L_{\text {mes }}$ and larger colour gamut $\left(L_{\text {mes }}+G V\right)$ under four lighting levels, are shown in Figures 4 and 5. Table 1 and Table 2 lists the peak wavelength and the ratios of LEDs. Also, their mesopic luminance ( $\left.L_{m e s}\right)$, gamut volume (GV), and S/P-ratio can be seen in Table 3 and Table 4.

Table 1 - The information of optimal white LED spectra (Philips Color Blast)

\begin{tabular}{|c|c|c|c|c|c|c|c|c|c|}
\hline \multirow{3}{*}{$\left(\mathrm{cd} / \mathrm{m}^{2}\right)$} & \multicolumn{9}{|c|}{ Peak Wavelength (nm) } \\
\hline & \multicolumn{3}{|c|}{ Lmes+GV } & \multicolumn{3}{|c|}{ Lmes } & \multicolumn{3}{|c|}{ GV } \\
\hline & Blue & Green & Red & Blue & Green & Red & Blue & Green & Red \\
\hline 10 & 445 & 545 & 620 & 445 & 570 & 605 & 435 & 535 & 645 \\
\hline 1 & 445 & 545 & 620 & 445 & 570 & 605 & 435 & 525 & 640 \\
\hline 0.3 & 455 & 540 & 620 & 480 & 570 & 605 & 435 & 525 & 640 \\
\hline 0.1 & 460 & 525 & 620 & 475 & 530 & 605 & 445 & 525 & 640 \\
\hline \multirow{3}{*}{$\left(\mathrm{cd} / \mathrm{m}^{2}\right)$} & \multicolumn{9}{|c|}{ Ratio } \\
\hline & \multicolumn{3}{|c|}{ Lmes+GV } & \multicolumn{3}{|c|}{ Lmes } & \multicolumn{3}{|c|}{ GV } \\
\hline & Blue & Green & Red & Blue & Green & Red & Blue & Green & Red \\
\hline 10 & 0.4 & 0.6 & 0.8 & 0.1 & 0.6 & 1.0 & 0.8 & 0.5 & 1.0 \\
\hline 1 & 0.4 & 0.6 & 0.8 & 0.1 & 0.6 & 1.0 & 0.4 & 0.3 & 0.5 \\
\hline 0.3 & 0.6 & 0.7 & 0.8 & 0.1 & 0.5 & 1.0 & 0.4 & 0.3 & 0.5 \\
\hline 0.1 & 0.4 & 0.5 & 0.6 & 0.1 & 0.2 & 0.7 & 0.5 & 0.4 & 0.6 \\
\hline
\end{tabular}



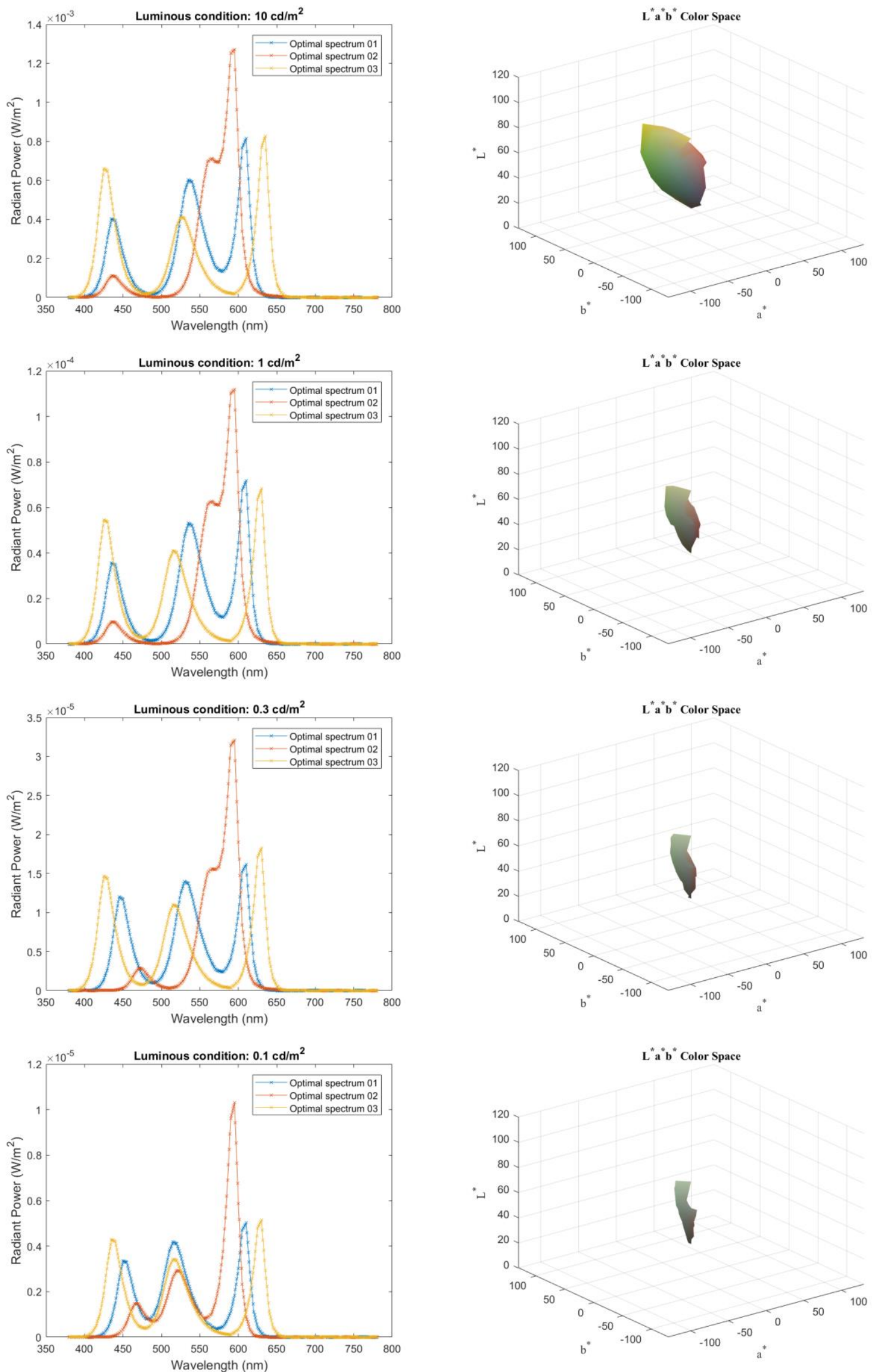

Figure 4 - Illustration of SPDs (left) and colour gamut (right) of SPD (Lmes+GV) under $10 \mathrm{~cd} / \mathrm{m}^{2}$, $1 \mathrm{~cd} / \mathrm{m}^{2}, 0.3 \mathrm{~cd} / \mathrm{m}^{2}$ and $0.1 \mathrm{~cd} / \mathrm{m}^{2}$ luminance levels (Philips Color Blast) 
For maximizing the $L_{\text {mes, }}$ the optimal spectra derived from Philips Color Blast and Gaussianlike function with similar peak power density ratio of blue, green and red LEDs are recommended for $10 \mathrm{~cd} / \mathrm{m}^{2}, 1 \mathrm{~cd} / \mathrm{m}^{2}$ and $0.3 \mathrm{~cd} / \mathrm{m}^{2}$ whereas the SPD with higher S/P-ratio for $0.1 \mathrm{~cd} / \mathrm{m}^{2}$ is different. The optimal spectra with higher S/P-ratio and CCT can improve the colour perception, especially under dim lighting conditions. Furthermore, a trade-off between mesopic luminance and colour gamut was quite evident.

Table 2 - The information of optimal white LED spectra (Gaussian-like function)

\begin{tabular}{|c|c|c|c|c|c|c|c|c|c|}
\hline \multirow{3}{*}{$\left(\mathbf{c d} / \mathbf{m}^{2}\right)$} & \multicolumn{9}{|c|}{ Peak Wavelength (nm) } \\
\cline { 2 - 11 } & Blue & Green & Red & Blue & Green & Red & Blue & Green & Red \\
\cline { 2 - 11 } & 445 & 555 & 610 & 455 & 570 & 605 & 435 & 535 & 625 \\
\hline 10 & 445 & 540 & 610 & 455 & 570 & 605 & 435 & 535 & 625 \\
\hline 1 & 460 & 540 & 615 & 470 & 570 & 610 & 435 & 535 & 625 \\
\hline 0.3 & 475 & 525 & 615 & 465 & 525 & 605 & 455 & 525 & 625 \\
\hline 0.1 & \multicolumn{9}{|c|}{ Ratio } \\
\hline \multirow{3}{*}{$\left(\mathbf{c d} / \mathbf{m}^{2}\right)$} & \multicolumn{7}{|c|}{ Lmes+GV } & \multicolumn{7}{|c|}{ Lmes } \\
\cline { 2 - 11 } & Blue & Green & Red & Blue & Green & Red & Blue & Green & Red \\
\hline 10 & 0.3 & 0.5 & 0.8 & 0.1 & 0.5 & 0.9 & 0.6 & 0.4 & 1.0 \\
\hline 1 & 0.2 & 0.3 & 0.6 & 0.1 & 0.5 & 0.9 & 0.6 & 0.4 & 1.0 \\
\hline 0.3 & 0.4 & 0.5 & 0.9 & 0.2 & 0.8 & 1.0 & 0.6 & 0.4 & 1.0 \\
\hline 0.1 & 0.5 & 0.4 & 0.7 & 0.2 & 0.3 & 0.6 & 0.5 & 0.4 & 0.9 \\
\hline
\end{tabular}



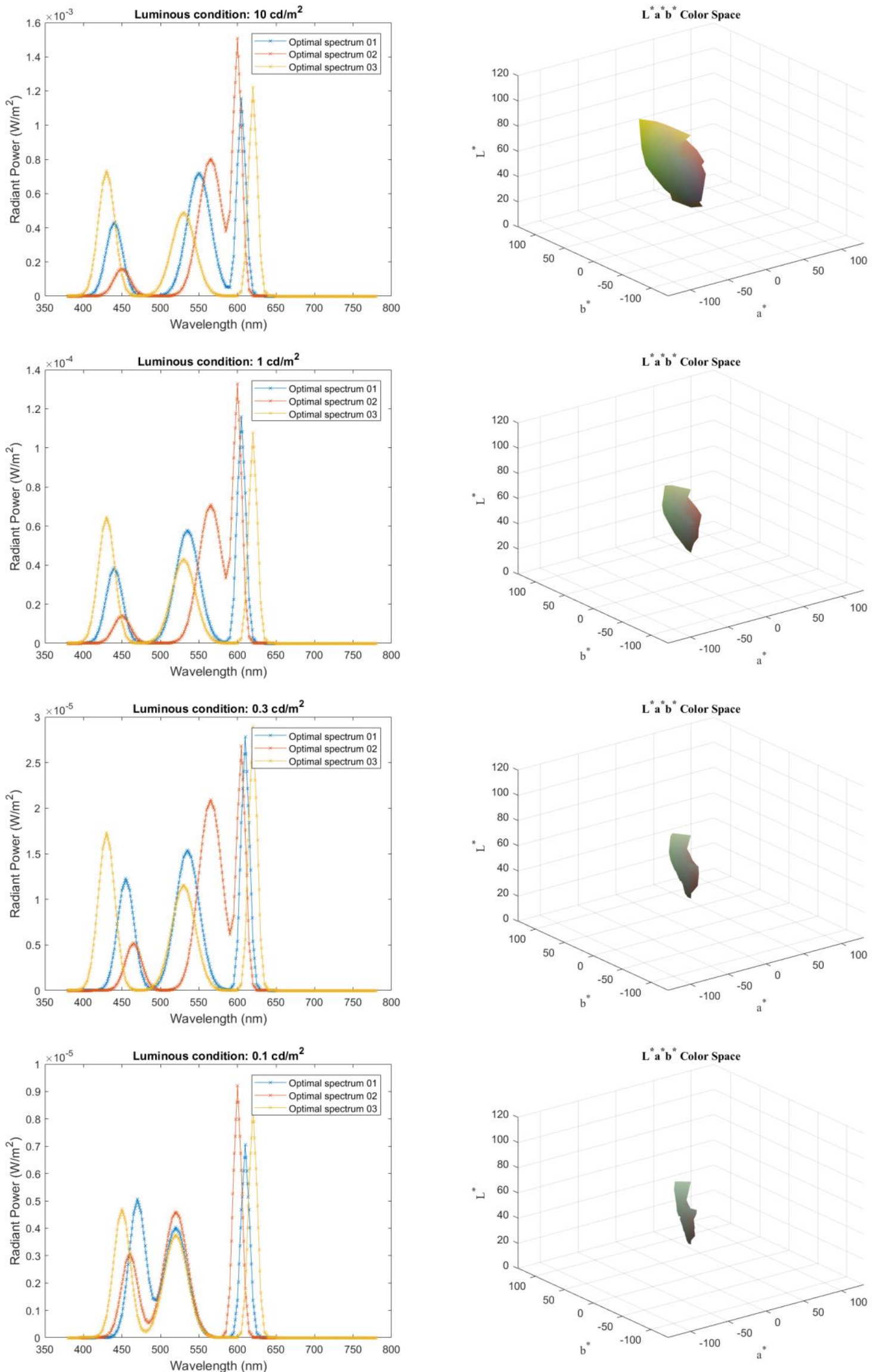

Figure 5 - Illustration of SPDs (left) and colour gamut (right) of SPD (Lmes+GV) under $10 \mathrm{~cd} / \mathrm{m}^{2}$, $1 \mathrm{~cd} / \mathrm{m}^{2}, 0.3 \mathrm{~cd} / \mathrm{m}^{2}$ and $0.1 \mathrm{~cd} / \mathrm{m}^{2}$ luminance levels (Gaussian-like function) 
Table 3 - Mesopic luminance ( $L_{m e s}$ ), gamut volume (GV) and S/P-ratio of optimal spectra (Philips Color Blast)

\begin{tabular}{|c|c|c|c|c|c|c|c|c|c|}
\hline \multirow{2}{*}{$\left(\mathbf{c d} / \mathbf{m}^{\mathbf{2}}\right)$} & \multicolumn{3}{|c|}{ Predicted Lmes } & \multicolumn{3}{c|}{ Gamut volume $\left(\mathbf{x 1 0} \mathbf{3}^{\mathbf{3}}\right.$} & \multicolumn{3}{c|}{ S/P-ratio } \\
\cline { 2 - 11 } & Lmes+GV & Lmes & GV & Lmes+GV & Lmes & GV & Lmes+GV & Lmes & GV \\
\hline 10 & 22.36 & 29.36 & 13.23 & 90.79 & 24.80 & 128.83 & 1.55 & 0.65 & 2.26 \\
\hline 1 & 2.03 & 2.55 & 1.33 & 17.30 & 3.97 & 29.52 & 1.55 & 0.65 & 2.68 \\
\hline 0.3 & 0.57 & 0.66 & 0.41 & 7.48 & 1.12 & 15.24 & 2.02 & 0.71 & 2.68 \\
\hline 0.1 & 0.18 & 0.19 & 0.15 & 3.06 & 1.00 & 4.59 & 2.70 & 1.39 & 2.95 \\
\hline
\end{tabular}

Table 4 - Mesopic luminance ( $L_{\text {mes }}$ ), gamut volume (GV) and S/P-ratio of optimal spectra (Gaussian-like function)

\begin{tabular}{|c|c|c|c|c|c|c|c|c|c|}
\hline \multirow{2}{*}{$\left(\mathrm{cd} / \mathrm{m}^{2}\right)$} & \multicolumn{3}{|c|}{ Predicted $L_{\text {mes }}$} & \multicolumn{3}{|c|}{ Gamut volume $\left(\times 10^{3}\right)$} & \multicolumn{3}{|c|}{ S/P-ratio } \\
\hline & $\mathrm{Lmes}_{\mathrm{m}} \mathrm{GV}$ & $L_{\text {mes }}$ & GV & $\mathrm{Lmes}_{\mathrm{m}} \mathrm{GV}$ & $L_{\text {mes }}$ & GV & $\mathrm{L}_{\mathrm{mes}}+\mathrm{GV}$ & Lmes & GV \\
\hline 10 & 24.31 & 28.42 & 15.05 & 80.78 & 33.78 & 134.35 & 1.25 & 0.71 & 2.11 \\
\hline 1 & 2.02 & 2.47 & 1.44 & 18.65 & 5.82 & 30.26 & 1.70 & 0.71 & 2.11 \\
\hline 0.3 & 0.57 & 0.65 & 0.43 & 7.80 & 2.923 & 14.81 & 2.05 & 0.86 & 2.11 \\
\hline 0.1 & 0.18 & 0.19 & 0.16 & 3.28 & 2.47 & 5.00 & 3.40 & 2.48 & 3.07 \\
\hline
\end{tabular}

By applying more simulated spectral data, the optimization method of the trichromatic white LED spectrum can well find the solutions to raise the mesopic luminance ( $L$ mes) and enlarge the colour gamut volume (GV). The results show that the green component is a benefit for mesopic luminance, and the red and blue LEDs influence the range of colour gamut most. Under $0.1 \mathrm{~cd} / \mathrm{m}^{2}$ lighting condition, the green channel of the optimal spectrum producing the highest mesopic luminance shifts to the shorter wavelength position following the conversion of mesopic luminous efficiency from photopic to scotopic vision. Figures 6 and 7 are the simulated images of mesopic colour reproduction using the optimal spectra. The information of Duv, CCT, and colour rendering properties (CRI, $R_{f}$ and, $R_{g}$ ) are listed in Table 5 . The optimal spectra $\left(L_{m e s}+G V\right)$ can also present the acceptable colour performance for mesopic conditions. However, the majority of the white LED spectrum is optimized based on luminous efficiency and colour rendering index mostly in the photopic vision, which shows a wider colour gamut than in mesopic vision. For dim lighting conditions, the conventional colour rendering properties such as CRI and IES TM-30-15 cannot express the colour variation with the decreasing of illuminance.

Table 5 - CCT, Duv and colour rendering properties of ( $\left.L_{\text {mes }}+G V\right)$ white LED spectra

\begin{tabular}{|c|c|c|c|c|c|c|c|c|c|c|}
\hline \multirow{2}{*}{$\left(\mathbf{c d} / \mathbf{m}^{2}\right)$} & \multicolumn{4}{|c|}{ Philips Color Blast } & \multicolumn{4}{c|}{ Gaussian-like function } \\
\cline { 2 - 12 } & CCT $(\mathrm{K})$ & Duv & CRI & $\mathrm{R}_{\mathrm{f}}$ & $\mathrm{R}_{\mathrm{g}}$ & $\mathrm{CCT}(\mathrm{K})$ & Duv & $\mathrm{CRI}$ & $\mathrm{R}_{\mathrm{f}}$ & $\mathrm{R}_{\mathrm{g}}$ \\
\hline 10 & 4,579 & 0.0162 & 68 & 66 & 97 & 4,332 & 0.0167 & 49 & 47 & 84 \\
\hline 1 & 4,579 & 0.0162 & 68 & 66 & 97 & 4,770 & 0.0183 & 72 & 68 & 102 \\
\hline 0.3 & 5,977 & 0.0145 & 77 & 75 & 99 & 5,643 & 0.0149 & 82 & 77 & 104 \\
\hline 0.1 & 6,665 & 0.0145 & 81 & 76 & 101 & 8,023 & 0.0185 & 59 & 53 & 89 \\
\hline
\end{tabular}


(a)

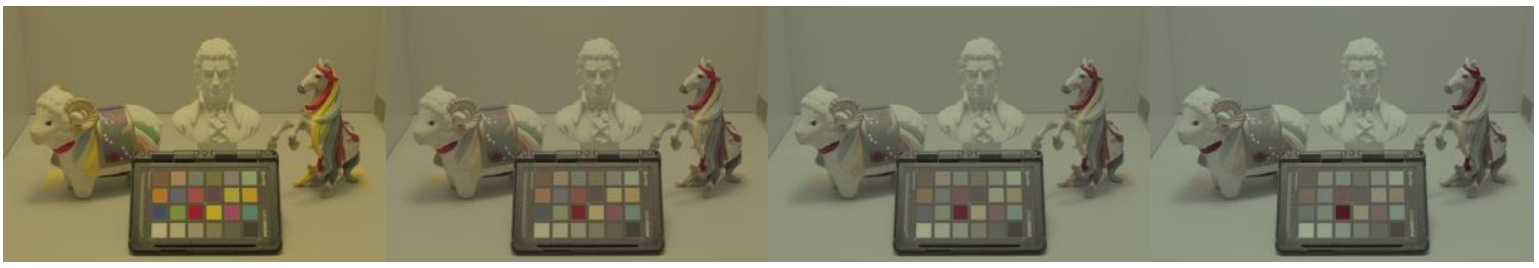

(b)

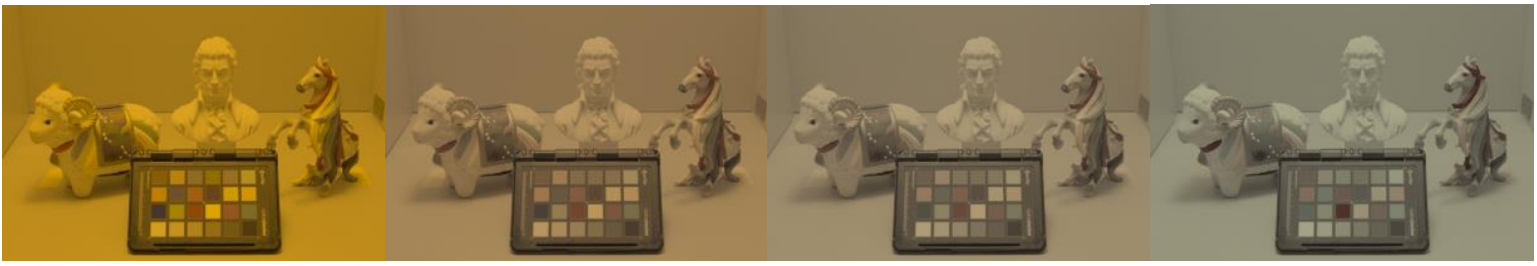

(c)

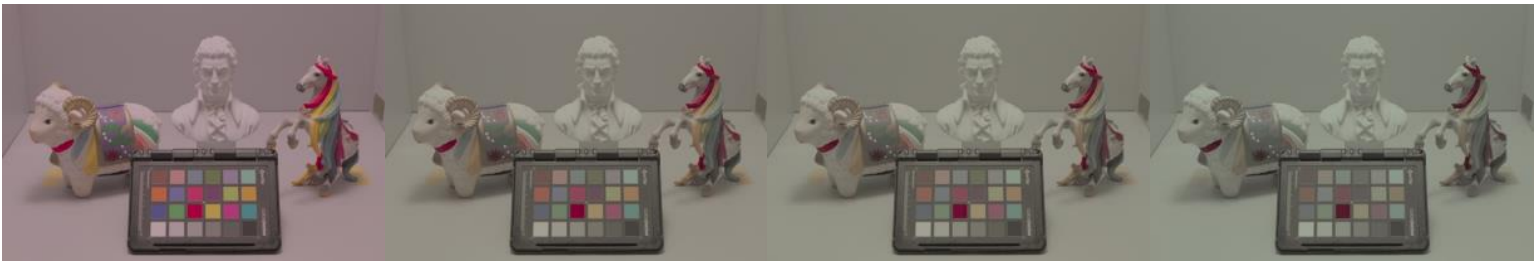

Figure 6 - Simulated images of optimal spectra (Philips Color Blast) under four lighting levels (left to right: $10 \mathrm{~cd} / \mathrm{m}^{2}, 1 \mathrm{~cd} / \mathrm{m}^{2}, 0.3 \mathrm{~cd} / \mathrm{m}^{2}$ and $0.1 \mathrm{~cd} / \mathrm{m}^{2},(\mathrm{a}): L_{\text {mes }}+\mathrm{GV}$ (b): $L_{\text {mes }}(\mathrm{c}): \mathrm{GV}$ )

(a)

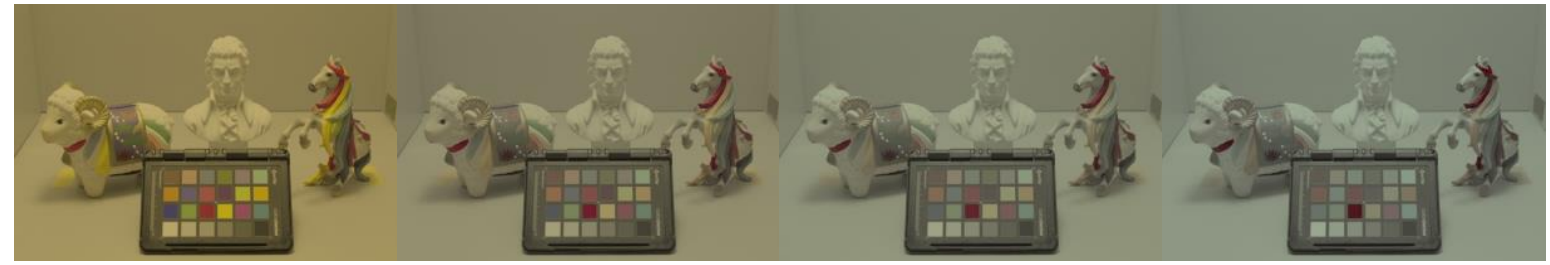

(b)

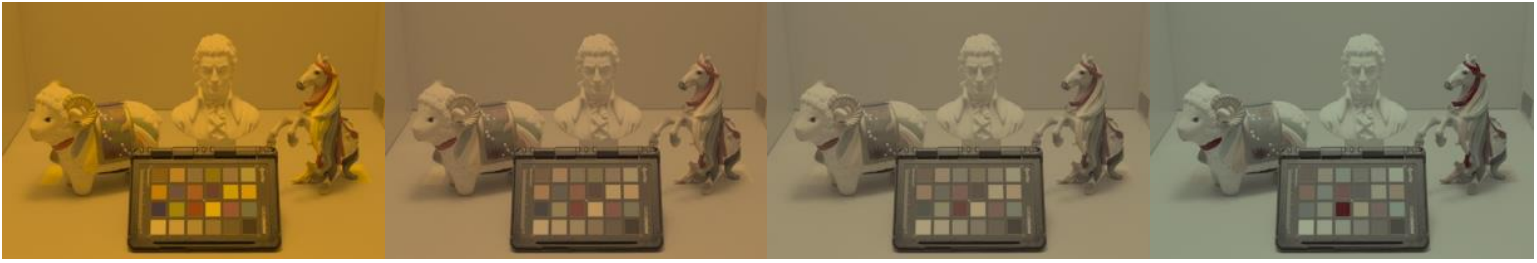

(c)

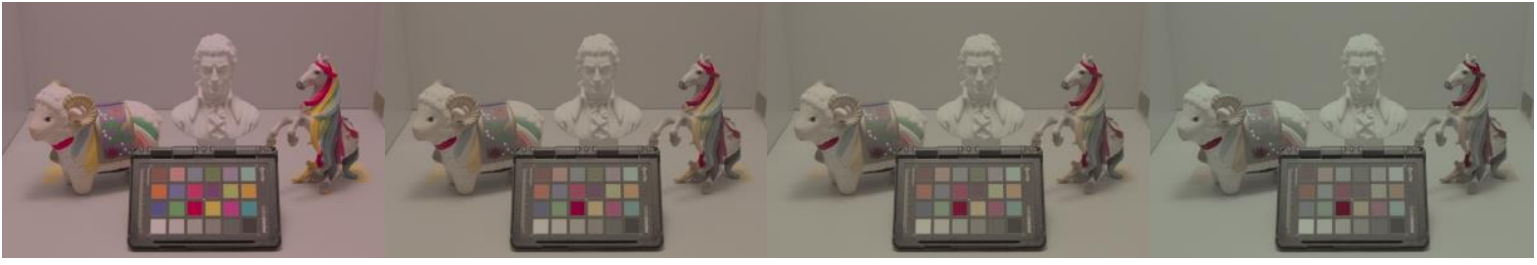

Figure 7 - Simulated images of optimal spectra (Gaussian-like function) under four lighting levels (left to right: $10 \mathrm{~cd} / \mathrm{m}^{2}, 1 \mathrm{~cd} / \mathrm{m}^{2}, 0.3 \mathrm{~cd} / \mathrm{m}^{2}$ and $\left.0.1 \mathrm{~cd} / \mathrm{m}^{2},(\mathrm{a}): L_{\text {mes }}+G V(b): L_{m e s}(\mathrm{c}): \mathrm{GV}\right)$ 

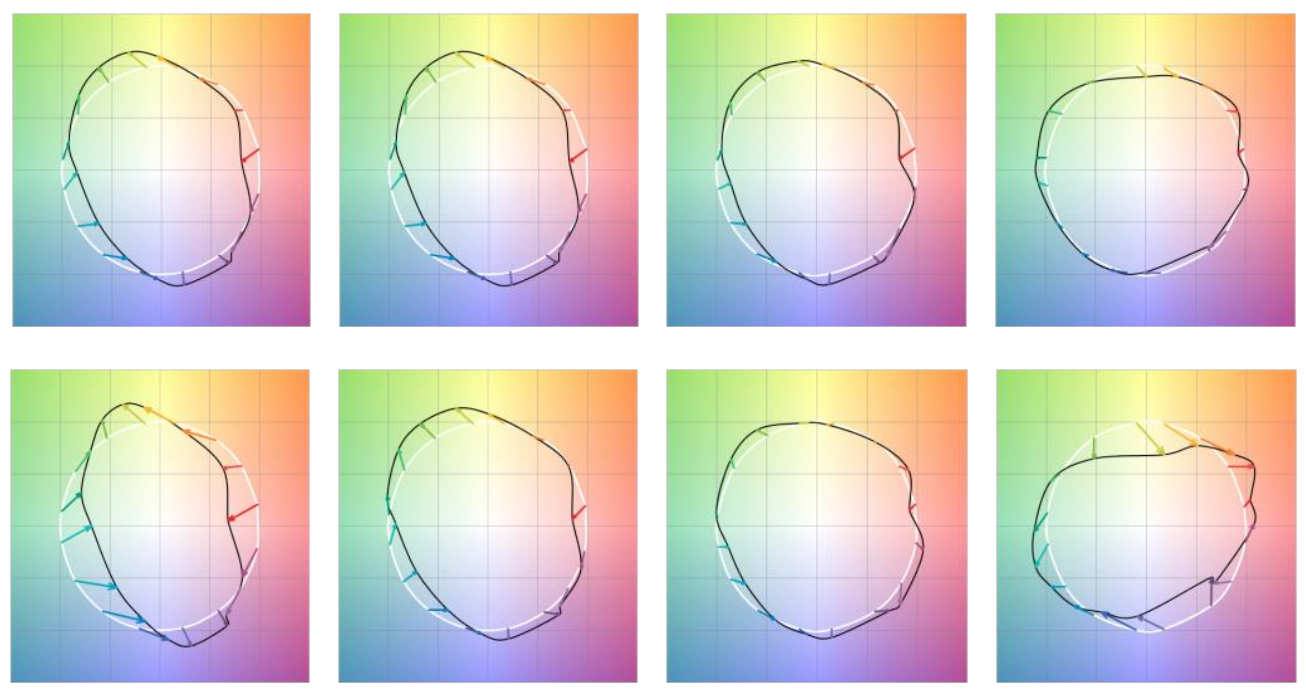

\section{Figure 8 - Colour Vector Graphic of optimal trichromatic white LED (Lmes+GV) (top: Philips Color Blast, bottom: Gaussian-like function) for four lighting levels (left to right: $10 \mathrm{~cd} / \mathrm{m}^{2}$, $1 \mathrm{~cd} / \mathrm{m}^{2}, 0.3 \mathrm{~cd} / \mathrm{m}^{2}$ and $0.1 \mathrm{~cd} / \mathrm{m}^{2}$ )}

\section{Conclusions}

In the study, the RGB-LED spectra of Philips Color Blast and simulated by applying Gaussianlike function are tested for better knowing the characteristics of white LED spectra. To obtain mesopic luminous, S/P-ratio of a light source and its photopic luminous are needed. The theoretical simulation uses the mesopic colour appearance model to investigate the gamut volume under optimal white LED spectra. An optimization theory based on the modified MOVE-model and a mesopic colour appearance model is applied to optimize trichromatic white LED spectra for lighting design. Three kinds of white LED spectra with acceptable Duv are recommended for improving visual perception for each luminance level from two datasets. From the simulated results, the SPDs significantly influences the predicted mesopic luminance and 3D colour gamut. The predicted mesopic luminance increases for a blue LED by shifting the peak wavelength to a long position. Besides, the higher power density ratio of blue and red LED contributes to better performance of colour gamut. Comparing our results with the previous study, the optimal spectra we proposed can produce higher mesopic luminance but not for colour gamut volume because of the trade-off between these two features. However, the 24 optimal spectra are the results of local optimization. For future work, the global optimization for trichromatic white LED spectra can be discussed with parallel computing, and a visual experiment is necessary to verify the effect of lighting applications.

\section{References}

Eloholma, M., Viikari, M., Halonen, L., Walkey, H., Goodman, T., Alferdinck, J. W. A. M., ... \& Várady, G. 2005. Mesopic models-from brightness matching to visual performance in night-time driving: a review. Lighting Res. Technol., 37(2), 155-173.

Rea, M. S., Bullough, J. D., Freyssinier-Nova, J. P., \& Bierman, A. 2004. A proposed unified system of photometry. Lighting Res. Technol., 36(2), 85-109.

Eloholma, M., \& Halonen, L. 2006. New model for mesopic photometry and its application to road lighting. Leukos, 2(4), 263-293.

Halonen, L., \& Puolakka, M. 2010. CIE and mesopic photometry. CIE NEWS, 1-2.

Viikari, M., Ekrias, A., Eloholma, M., \& Halonen, L. 2008. Modeling spectral sensitivity at low light levels based on mesopic visual performance. Clinical ophthalmology (Auckland, NZ), 2(1), 173. 
Zan, L., Lin, D., Zhong, P., \& He, G. 2016. Optimal spectra of white LED integrated with quantum dots for mesopic vision. Optics Express, 24(7), 7643-7653.

CIE 2010. CIE 191:2010. Recommended System for Mesopic Photometry Based on Visual Performance. Vienna: CIE.

Shin, J., Matsuki, N., Yaguchi, H., \& Shioiri, S. 2004. A color appearance model applicable in mesopic vision. Optical review, 11(4), 272-278.

Kaiser, P. K., \& Boynton, R. M. 1996. Human color vision.

Li, H. C., Huang, Y. N., \& Sun, P. L. 2018. A comparison of optimal white LED spectra under mesopic condition. Optics \& Photonics Taiwan International Conference.

Illuminating Engineering Society of North America. 2015. IES TM-30-15, IES Method for Evaluating Light Source Color Rendition. Illuminating Engineering Society of North America.

Li, H. C., Sun, P. L., \& Ronnier Luo, M. 2016. Optimization of White LED Spectrum under Mesopic Condition Based on 3D Color Gamut. 4th CIE Symposium on Color and Visual Appearance, Prague, Czech Republic. 